\title{
Fibrous Pseudotumor of the Tunica Vaginalis of the Scrotum: Is there a Typical Ultrasound Pattern?
}

\section{Introduction \\ $\nabla$}

The fibrous pseudotumor of the scrotum is a relatively rare benign condition that clinically often mimics malignancy. Consequently, the diagnosis is usually performed only after orchidectomy.

Our goal was to determine if there are any US features which are indicative of benignity in order to avoid excision of the testis based on these 3 cases.

\section{Case 1}

$\nabla$

A 42-year-old man presented to the surgical outpatient department for a left scrotal swelling associated with subacute tenderness and discomfort.

The lesion, initially painless, was already known by the patient and had gradually increased in size over a period of 2 years, with progressive tension and discomfort over the previous weeks. The only previous history was a radical prostatectomy for a prostatic carcinoma (stage pT2, Gleason score 3+4) 6 months before.

The physical examination revealed multiple firm to hard, irregular tender masses in the left scrotum, measuring from 1 to $3 \mathrm{~cm}$. These lesions were independent of the testis and were associated with an inflammatory appearance of the scrotum. Biological data and PSA level were normal.

Based on the pain and the suspicious clinical examination, a surgical exploration was decided upon. The testis was intact and could be preserved. The lesion surrounding the testis was constituted of masses that were adherent to the epididymis but attached to the tunica vaginalis. It could be totally removed after confirmation of the diagnosis by a frozen section.

The final histopathological diagnosis was a fibrous pseudo tumor of the tunica vaginalis.

Clinical and US follow-up examinations performed 6 months later were strictly normal.

\section{Case 2 \\ $\nabla$}

A 59-year-old man reported a progressive painless swelling of the right scrotum for 8 months. There was no previous history of a testicular abnormality. The patient underwent a transurethral resection for a prostatic hyperplasia one year ago.

On physical examination, multiple hard nodules were palpated simulating a large solid right testicular mass.

Because of the clinical suspicion of malignancy, a right orchidectomy was performed. The testis was uninvolved and surrounded by an irregular thickened tunica vaginalis.

The pathological analysis confirmed the diagnosis of a benign fibrous pseudo tumor of the tunica vaginalis.

\section{Case 3 \\ $\nabla$}

A 25-year-old man presented similar symptoms over a period of 2 years. The patient had no significant medical history. Clinical data were similar to those of case 1.

Surgical exploration revealed a multilobulated mass related to the left epididymis with involvement of the adjacent testicular tunica vaginalis. The masses, the left epididymis, and the vas deferens with the surrounding tissue were resected. The intact left testis was preserved. Final pathological diagnosis was of a benign fibrous pseudotumor of the tunica vaginalis.

\section{Results \\ $\nabla$}

Ultrasound examination ( $\odot$ Fig. 1-3a, b) revealed multiple round or ovoid, well circumscribed echoic and hypoechoic lesions, some of which partially calcified. The location was clearly extratesticular surrounding the normal testis and presumably involving the tunica vaginalis. A posterior acoustic shadowing coming from some nodules obscuring a part of the adjacent tissue was found in all our cases ( Fig. 1-3a, b). There was a moderate hydrocele in case 2 ( $\bullet$ Fig. $2 \mathbf{2 a}$ ). Color
Doppler showed minimal vascularity in a few echogenic nodules ( $\bullet$ Fig. 2 b, 3b). Macroscopic appearance were of multiple adjacent gray nodules ranging in size from 1 to $3 \mathrm{~cm}$ ( $\odot$ Fig. 1c).

At histology, all lesions were composed of dense fibrocollagenous stroma with chronic inflammation and no malignancy (॰ Fig. 1-3c).

\section{Discussion \\ $\nabla$}

The term of fibrous pseudotumor of the tunica vaginalis was first introduced in 1975 by Mostofi (FK. Mostofi et al. Atlas of Tumor Pathology. $2^{\text {nd }}$ Ed; 1973: 151154).

Even though its pathogenesis is not completely understood, this lesion is considered to represent a benign reactive fibrous proliferation in response to a previous infection or trauma; $30 \%$ of patients have a history of trauma or epididymoorchitis and $50 \%$ have a concomitant hydrocele. A non-negligible proportion of cases are probably idiopathic, such as in case 3.

Although this lesion is quite uncommon, accounting for $6 \%$ of all extratesticular abnormalities, it represents the third most common benign lesion after the spermatic cord lipoma and the epididymal adenomatoid tumor.

Approximately $85 \%$ of cases originate from the tunica vaginalis and the others from the epididymis, spermatic cord or the tunica albuginea.

The clinical presentation of our cases is similar to that encountered in the literature (M. L. Grebenc et al. Abdom Imaging 1995; 28: 379-380), (P. Germaine et al., J Ultrasound Med 2007; 26: 133-138).

The differential diagnosis includes some exceptional peritesticular lesions which, in contrast, presents as an isolated welldefined mass. Metastases involving the testes or the scrotum are unusual, occurring only in the context of a widely-disseminated malignancy (MA. Jones et al. Am J Surgpathol 1997; 21: 296-305).

Most cases in the literature report a radical orchidectomy as treatment, since the diagnosis was not suspected preoperatively and the clinical presentation more suggestive of a malignant tumour. Yet, since this is a benign condition, testicular-sparing surgery with complete local resection of the masses confirmed with a frozen section biopsy is the current treatment of choice. 

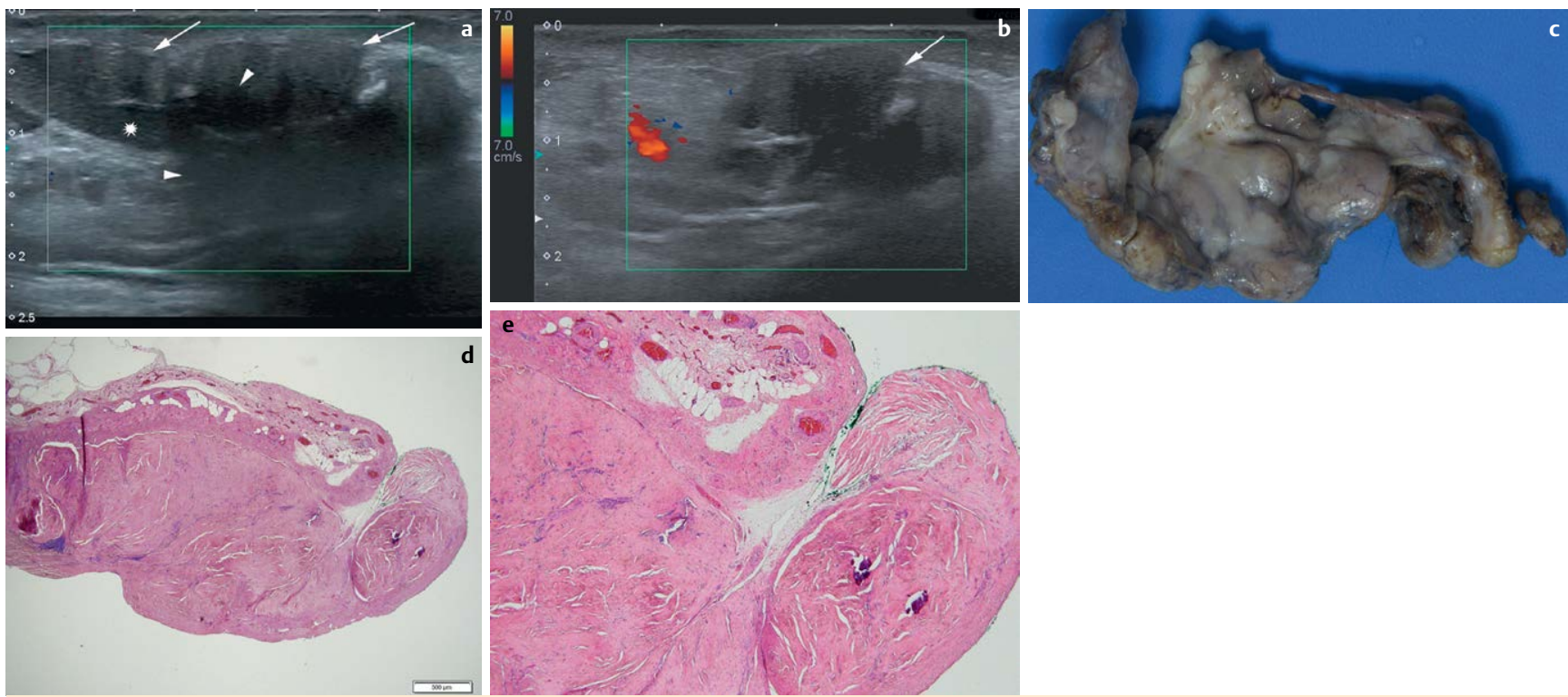

Fig. 1 Case 1, 42 yo male. a US sagittal image at the lower part of the scrotum. It shows multiple adjacent hypoechoic well marginated nodules ( $\uparrow$ ) attached to the tunica vaginalis and the epididymis $(\star)$. Some of them are calcified and others present an acoustic shadowing hiding the tail of the epididymis $(\Delta)$. $\mathbf{b}$ Color Doppler axial image at the lower part of the scrotum showing the absence of Doppler signal inside the nodules $(\uparrow)$. $\mathbf{c}$ Photograph of the gross specimen showing multiple regular white-grey nodules ranging from 1 to $3 \mathrm{~cm}$, placed side-by-side and attached to the tunica vaginalis, going up to $8 \mathrm{~cm}$ in their greatest length axis. $\mathbf{d}$ Histologic section (hematoxylin-eosin, original magnification $\times 2$ ) showing a dense fibrous tissue thickening of the tunica vaginalis, sometimes forming non-encapsulated nodules.
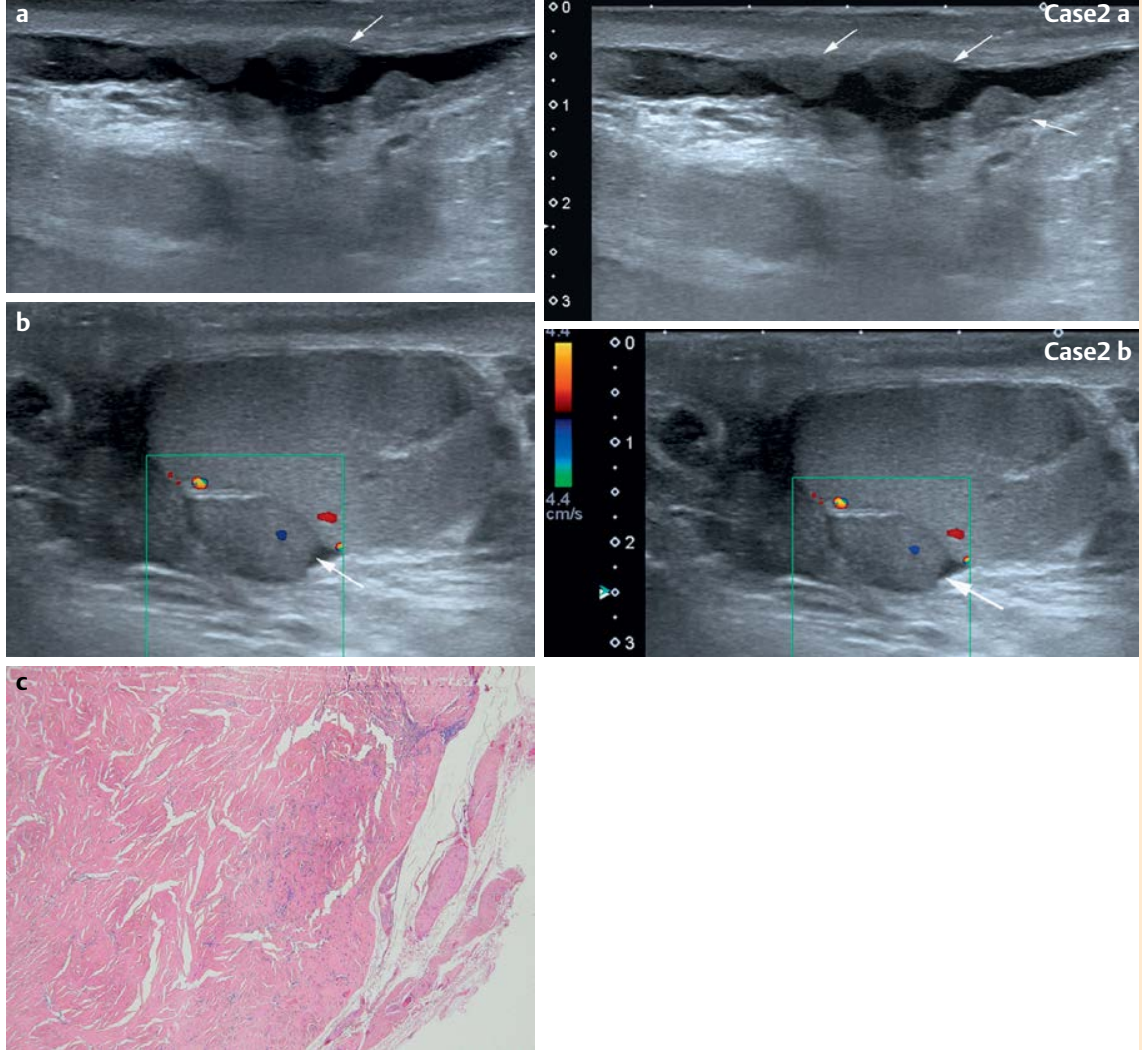

Several authors have reported the ultrasonographic appearance of this entity. However, none have confidently relied solely on the ultrasound findings without surgical exploration.

Based on the 3 cases described in this paper, we found that the constant acoustic

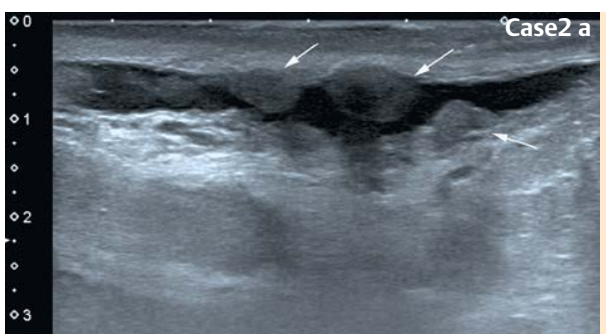

Fig. 2 Case 2, 59 yo male. a US sagittal image at the middle part of the scrotum. It shows multiple confluent hypoechoic nodules of varying size coming from the tunica vaginalis $(\uparrow)$. They are surrounded by a small quantity of hydrocele. Note the presence of acoustic shadowing coming from the largest nodule $(\uparrow)$. b Color Doppler sagittal image at the upper part of the scrotum. It shows clearly the integrity of the testis. One well-circumscribed echogenic nodule presents a sparse vascularity. Several other nodules are attached to the tunica vaginalis ( $\uparrow$ ). c Histologic section (hematoxylineosin, original magnification $\times 4$ ) of one of the small hypoechoic nodule shows a dense hyalinized collagen stroma with some scattered lymphocytes and plasmocytes due to chronic inflammation. 

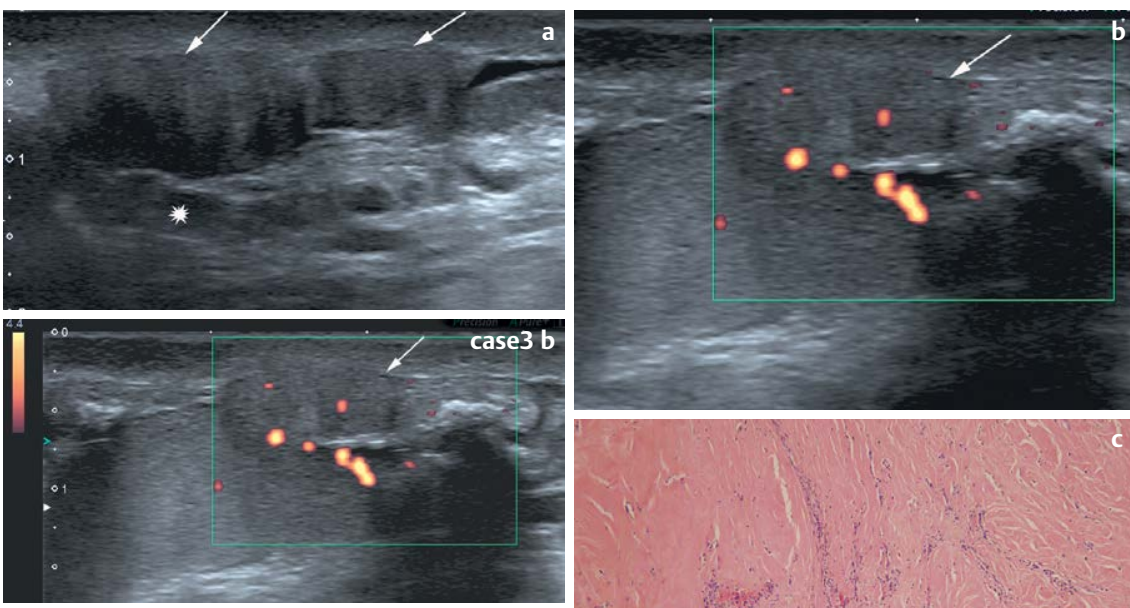

Fig. 3 Case 3, 25 yo male. a US sagittal image at the lower part of the scrotum shows multiple more or less hypoechoic masses arising from the tunica vaginalis surface, the scrotum wall ( $\uparrow$ ) and around the epididymis $(\star)$. Note also the acoustic shadowing hiding the inferior part of the testis. $\mathbf{b}$ Power Doppler of one large nodule shows moderate vascularization inside a more echoic nodule $(\uparrow)$. c Histologic section (hematoxylin-eosin, original magnification $\mathrm{x} 10$ ) shows abundant collagen tissue with poor cellularity but without atypia, some

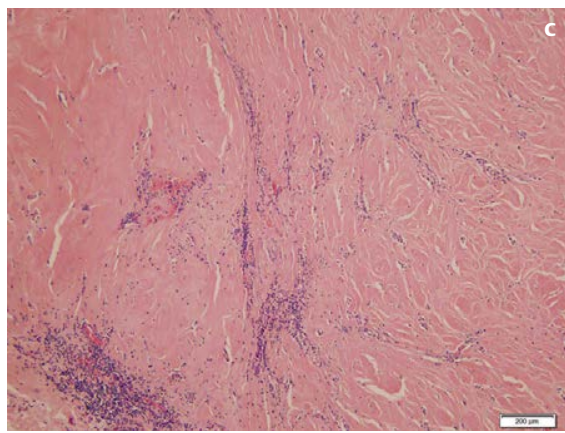
embedded inflammatory cells.

ated with significant acoustic shadowing and low to absent Doppler signal should lead the radiologist to the diagnosis of pseudotumor of the tunica vaginalis.

Although case reports with MRI are limited, some authors have reported a specific appearance with an extratesticular multiple nodular lesion exhibiting intermediate-to-low signal intensity on T1weighted and T2-weighted images, with little or no gadolinium enhancement. The hypo-intensity on T2-weighted images is probably also a consequence of the dense fibrous content of these lesions (FH. Cassidy et al. RadioGraphics 2010; 30: 665683).

In conclusion, there are specific ultrasonic appearances to suggest the diagnosis of a fibrous pseudotumour sufficient to a warrant a conservative approach.

\section{Catchwords}

$\nabla$

- This a rare benign entity which has a characteristic pattern on US examination.

- The radiologist should be aware of it to orientate towards conservative surgery.

\section{References}

1 Mostofi FK, Price EB. Tumors of the male genital system. In: Atlas of Tumor Pathology. $2^{\text {nd }} \mathrm{Ed}$, fascicle 8. Washington DC: Armed Forces Institute of Pathology; 1973: 151-154

2 Grebenc ML, Gorman JD, Sumida FK. Fibrous pseudotumor of the tunica vaginalis testis. Abdom Imaging 1995; 20: 379-380

3 Germaine P, Simerman LP. Fibrous Pseudotumor of the Scrotum. J Ultrasound Med 2007; 26: 133-138
4 Jones MA, Young RH, Scully $R$. Benign fibromatous tumors of the testis and paratesticular region: a report of 9 cases with a proposed classification of fibromatous tumors and tumor-like lesions. Am J Surg Pathol 1997; 21: 296-305

5 Cassidy FH, Ishioka KM, McMahon CJ et al. MR imaging of scrotal tumors and pseudotumors. RadioGraphics 2010; 30: 665-683

M. Ohana, V. Lindner, A. Labani, G. Alemann, H. Lang, C. Roy 